\title{
Production, spectroscopy and properties of heavy hadrons
}

Mario Galanti* for the ALICE, ATLAS, CMS, and LHCb Collaborations

University of Rochester

E-mail: mario.galanti@cern.ch

In this report I will describe the latest results of the ALICE, ATLAS, CMS, and LHCb experiments in the fields of production, spectroscopy, and properties of heavy hadrons. In particular, I will concentrate on measurements of quarkonium production cross sections, polarization, and mass, on measurements of production cross sections and lifetimes of open heavy flavors, on the recent observations of new states and decay modes, and on other searches for new and exotic hadrons.

Sixth Annual Conference on Large Hadron Collider Physics (LHCP2018)

4-9 June 2018

Bologna, Italy

${ }^{*}$ Speaker. 


\section{Introduction}

In this report I will describe the latest results of the ALICE [1], ATLAS [2], CMS [3], and $\mathrm{LHCb}[4]$ experiments in the fields of production, spectroscopy, and properties of heavy hadrons. In particular, I will concentrate on measurements of quarkonium production cross sections, polarization, and mass, on measurements of production cross sections and lifetimes of open heavy flavors, on the recent observations of new states and decay modes, and on other searches for new and exotic hadrons.

\section{Quarkonium production and properties}

\subsection{Quarkonium production and polarization measurements}

The production cross sections of heavy quarkonia at central and forward rapidity have been measured with $13 \mathrm{TeV}$ data by CMS [5] and LHCb [6], respectively. CMS used $2.3-2.7 \mathrm{fb}^{-1}$ of data to measure the single- and double-differential production cross sections times branching fractions of five $s$-wave states $(\mathrm{J} / \psi, \psi(2 \mathrm{~S})$, and $\mathrm{Y}(n \mathrm{~S})$, with $n=1,2,3)$ as a function of $p_{\mathrm{T}}$ and rapidity. Cross sections were compared with NRQCD [7] predictions, and ratios of $13 \mathrm{TeV}$ cross sections for different states and $13 / 7 \mathrm{TeV}$ ratios for the same state were also given. The measured cross sections for $\mathrm{J} / \psi$ and $\psi(2 \mathrm{~S})$ are shown, as an example, in Fig. 1, left. LHCb used $277 \mathrm{pb}^{-1}$ of data to measure the single- and double-differential cross sections times branching fractions of the three $\mathrm{Y}(n \mathrm{~S})$ states. LHCb also measured the ratios of $13 \mathrm{TeV}$ cross sections for different $\mathrm{Y}(n \mathrm{~S})$ states and the $13 / 8 \mathrm{TeV}$ ratio for the same state. The $\mathrm{Y}(n \mathrm{~S})$ cross sections and the $\mathrm{Y}(2 \mathrm{~S}) / \mathrm{Y}(1 \mathrm{~S})$ cross section ratio are shown in the right part of Fig. 1. In general, the picture given by the two experiment is consistent. All cross sections measured by CMS and LHCb are in good agreement with the NRQCD predictions, and both experiments measure an increase of the ratios with $p_{\mathrm{T}}$, whose size depends on the type of particle and on the rapidity region probed. These measurements confirm earlier results by the ATLAS experiment at 7 and $8 \mathrm{TeV}$ [8].

Quarkonium polarization measurements are complementary to the cross section ones, as they allow to probe the quarkonium production process and to test the available hadronization models (e.g. color-octet [7] vs. color-singlet [9]). The polarization of $\mathrm{J} / \psi$ as a function of $p_{\mathrm{T}}$ and $y$ was recently measured by ALICE [10] on $8 \mathrm{TeV}$ pp data in the kinematic range $2<p_{\mathrm{T}}<15 \mathrm{GeV}$ and $2.5<y<4.0$. The longitudinal and transverse polarization parameters $\lambda_{\theta}, \lambda_{\varphi}$, and $\lambda_{\theta \varphi}$ were extracted in two different reference frames (Collins-Soper and Helicity), and the frame-independent parameter $\tilde{\lambda}$ was also computed. All parameters were found to have values compatible with zero in the full kinematic range probed, with no model being able to describe all the experimental results. This measurement follows a trend of null polarizations found in the past by LHC experiments both at central and at forward rapidity.

\subsection{Observation of $\chi_{\mathrm{b} 1,2}(3 \mathrm{P})$ mass split}

The CMS experiment recently reported about the first observation of the mass split in the $\chi_{\mathrm{b} 1}(3 \mathrm{P})-\chi_{\mathrm{b} 2}(3 \mathrm{P}) \mathrm{P}$-wave quarkonium doublet [11]. The $\chi_{\mathrm{b}}(3 \mathrm{P})$ state was first discovered by ATLAS in 2012 [12], however, no experiment has been able to probe its internal structure so far. CMS looked at the $\chi_{\mathrm{b} 1,2}(3 \mathrm{P}) \rightarrow \mathrm{Y}(3 \mathrm{~S}) \gamma$ decay, with $\gamma$ converting into $\mathrm{e}^{+} \mathrm{e}^{-}$inside the tracker volume, 

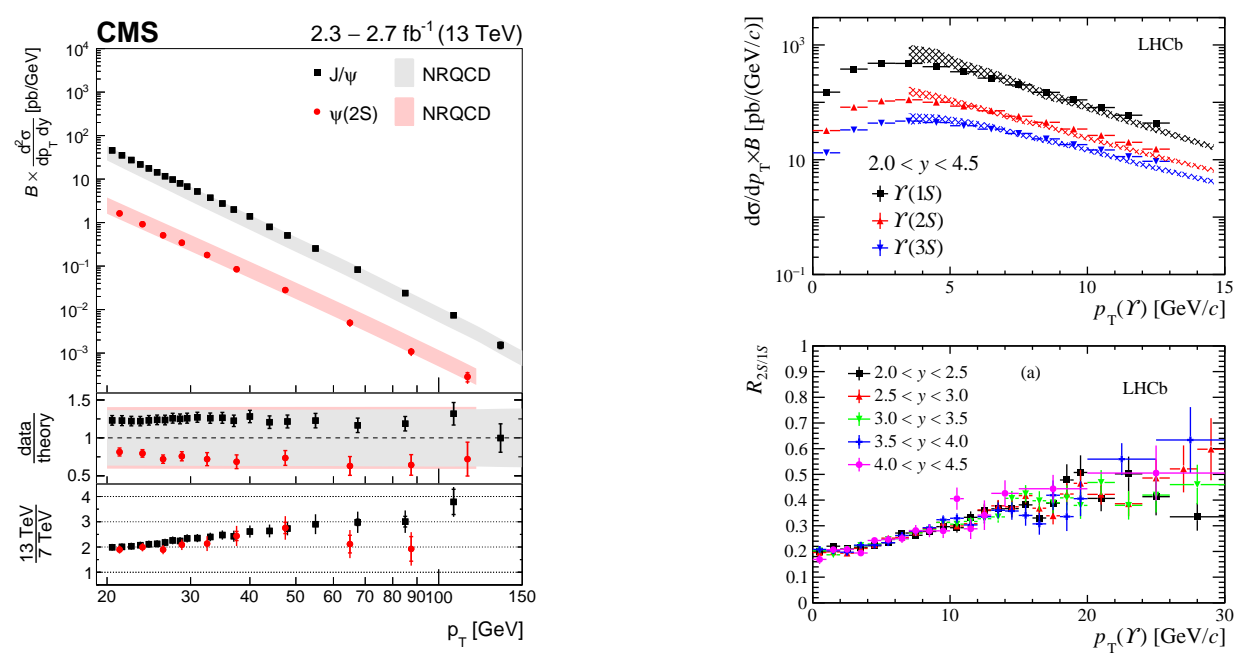

Figure 1: Left: the $\mathrm{J} / \psi$ and $\psi(2 \mathrm{~S})$ cross sections at $13 \mathrm{TeV}$ measured by CMS [5] at central rapidity as a function of $p_{\mathrm{T}}$. Bottom panels show the data/theory and the $13 / 7 \mathrm{TeV}$ differential ratios. Top right: the $\mathrm{Y}(n \mathrm{~S})$ cross sections at $13 \mathrm{TeV}$ measured by $\mathrm{LHCb}[6]$ at forward rapidity as a function of $p_{\mathrm{T}}$. Bottom right: the $\mathrm{Y}(2 \mathrm{~S}) / \mathrm{Y}(1 \mathrm{~S})$ cross section ratio measured by $\mathrm{LHCb}[6]$ as a function of $p_{\mathrm{T}}$ and rapidity. Color bands in figures show the NRQCD predictions.

in order to achieve an excellent mass resolution $(2.2 \mathrm{MeV})$ and observe the doublet separation with more than $5 \sigma$ significance. The observation plot can be seen in Fig. 2. The masses of the two states are measured to be, respectively, $M_{1}=10513.42 \pm 0.41$ (stat) \pm 0.18 (syst) MeV and $M_{2}=10524.02 \pm 0.57$ (stat) \pm 0.18 (syst) $\mathrm{MeV}$, with a mass splitting $\Delta M=10.60 \pm 0.64$ (stat) \pm 0.17 (syst) MeV. This measurement significantly constrains theoretical predictions, now giving mass splits in the range $[-2,+18] \mathrm{MeV}$.

\section{Open heavy flavor production and properties}

\subsection{Production measurements}

The production cross sections of $\Lambda_{\mathrm{c}}^{+}$and $\Xi_{\mathrm{c}}^{0}$ at central rapidity were measured by ALICE on pp data at $7 \mathrm{TeV}\left(\Lambda_{\mathrm{c}}^{+}\right.$and $\left.\Xi_{\mathrm{c}}^{0}\right)$ and $\mathrm{pPb}$ data at $5.02 \mathrm{TeV}\left(\Lambda_{\mathrm{c}}^{+}\right.$only) [13, 14]. For both states, the differential cross sections as a function of $p_{\mathrm{T}}$ are given. In addition, also the $\Lambda_{\mathrm{c}}^{+} / \mathrm{D}^{0}$ and $\Xi_{\mathrm{c}}^{0} / \mathrm{D}^{0}$ cross section ratios are given (the latter can be seen in Fig. 3). Results are compared with the expectations from perturbative QCD and from MC generators. None of the tested models is found to well reproduce data, underestimating the $\Lambda_{\mathrm{c}}^{+}$cross sections and both cross section ratios.

The charge asymmetry in the prompt production of $\mathrm{D}_{\mathrm{s}}^{ \pm}$mesons is defined as:

$$
A_{\mathrm{P}}\left(\mathrm{D}_{\mathrm{s}}^{+}\right)=\frac{\sigma\left(\mathrm{D}_{\mathrm{s}}^{+}\right)-\sigma\left(\mathrm{D}_{\mathrm{s}}^{-}\right)}{\sigma\left(\mathrm{D}_{\mathrm{s}}^{+}\right)+\sigma\left(\mathrm{D}_{\mathrm{s}}^{-}\right)} .
$$

This observable allows to probe experimentally the non-perturbative hadronization process and is an essential input for $\mathrm{D}_{\mathrm{S}} \mathrm{CP}$-violating measurements. $\mathrm{LHCb}$ recently released a measurement of $A_{\mathrm{P}}\left(\mathrm{D}_{\mathrm{S}}^{+}\right)$based on $3 \mathrm{fb}^{-1}$ of pp data at 7 and $8 \mathrm{TeV}$ [15]. The inclusive asymmetry measured by LHCb is $A_{\mathrm{P}}\left(\mathrm{D}_{\mathrm{s}}^{+}\right)=(-0.52 \pm 0.13$ (stat) \pm 0.10 (syst) $) \%$, corresponding to a $3.3 \sigma$ deviation from 
zero. The double-differential asymmetry as a function of the $\mathrm{D}_{\mathrm{s}} p_{\mathrm{T}}$ and $y$ is also measured. No significant kinematic dependence is seen. The experimental measurement is not compatible with the results from PYTHIA 8.1 [16], which predicts a positive asymmetry and a significant kinematic dependence. It is however in agreement with an older result at $\sqrt{s}=7 \mathrm{TeV}$ from $\mathrm{LHCb}$ [17].

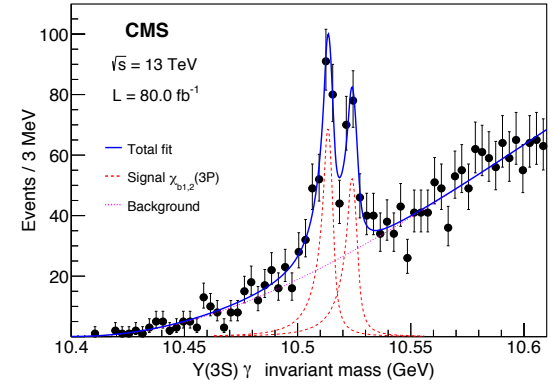

Figure 2: The $\chi_{\mathrm{b} 1,2}(3 \mathrm{P}) \rightarrow \mathrm{Y}(3 \mathrm{~S}) \gamma$ invariant mass measured by CMS on the full Run- 2 dataset. The two peaks corresponding to $\chi_{\mathrm{b} 1}(3 \mathrm{P})$ and $\chi_{\mathrm{b} 2}(3 \mathrm{P})$ are clearly visible [11].

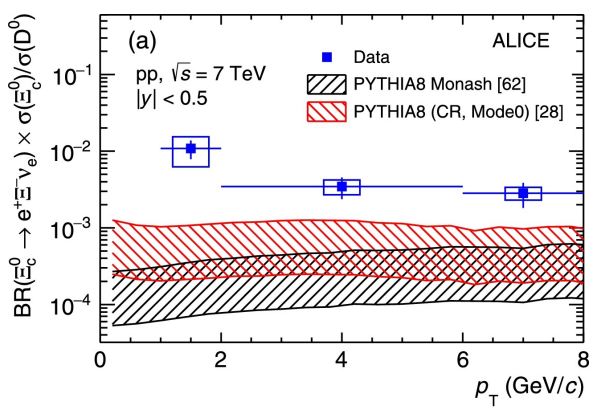

Figure 3: The $\Xi_{c}^{0} / D^{0}$ cross section ratio as a function of $p_{\mathrm{T}}$ measured by ALICE on pp data at $\sqrt{s}=$ $7 \mathrm{TeV}$ [14]. The predictions from two models are also shown.

\subsection{Lifetime measurements}

The lifetimes of four B hadrons $\left(\mathrm{B}^{0}, \mathrm{~B}_{\mathrm{s}}^{0}, \Lambda_{\mathrm{b}}^{0}\right.$, and $\left.\mathrm{B}_{\mathrm{c}}^{+}\right)$were measured by CMS with $8 \mathrm{TeV} \mathrm{pp}$ data [18], using the decay modes $\mathrm{B}^{0} \rightarrow \mathrm{J} / \psi \mathrm{K}^{\star}(892), \mathrm{B}^{0} \rightarrow \mathrm{J} / \psi \mathrm{K}_{\mathrm{S}}, \mathrm{B}_{\mathrm{s}}^{0} \rightarrow \mathrm{J} / \psi \phi(1020)^{0}, \mathrm{~B}_{\mathrm{s}}^{0} \rightarrow$ $\mathrm{J} / \psi \pi^{+} \pi^{-}, \Lambda_{\mathrm{b}}^{0} \rightarrow \mathrm{J} / \psi \Lambda^{0}$, and $\mathrm{B}_{\mathrm{c}}^{+} \rightarrow \mathrm{J} / \psi \pi^{+}$. All lifetime measurements are absolute, except for the $\mathrm{B}_{\mathrm{c}}^{+}$case, where the measurement is made with respect to the $\mathrm{B}^{+}$lifetime. The results are summarized in Table 1. CMS results are compared with the values in PDG 2016 [19]: they are found in agreement with the world averages, with a comparable or better precision. The two decays used to measure the $\mathrm{B}_{\mathrm{s}}^{0}$ lifetime have contributions from different amounts of heavy and light eigenstates, which allows to use them as input to extract the $\mathrm{B}_{\mathrm{sL}}^{0}$ lifetime. This is found to be $\mathrm{c} \tau_{\mathrm{B}_{\mathrm{sL}}^{0}}=420.4 \pm 6.1$ (stat + syst) $\mu \mathrm{m}$, in agreement with the PDG average of $423.6 \pm 1.8 \mu \mathrm{m}$.

Another recent lifetime measurement comes from LHCb and concerns the doubly-charmed $\Xi_{\mathrm{cc}}^{++}$baryon. This state was discovered by LHCb in 2017 in the $\Xi_{\mathrm{cc}}^{++} \rightarrow \Lambda_{\mathrm{c}}^{+} \mathrm{K}^{-} \pi^{+} \pi^{+}$decay mode [20]. A new LHCb analysis done on $1.7 \mathrm{fb}^{-1}$ of $13 \mathrm{TeV}$ data now measures the $\Xi_{\mathrm{cc}}^{++}$lifetime in the same decay mode, with respect to the $\Lambda_{\mathrm{b}}^{0}$ lifetime measured using the $\Lambda_{\mathrm{b}}^{0} \rightarrow \Lambda_{\mathrm{c}}^{+} \pi^{-} \pi^{+} \pi^{-}$ decay [21]. Given that the topologies of the two decays are similar, most systematic uncertainties cancel out in the ratio. The lifetime is found to be $\tau\left(\Xi_{\mathrm{cc}}^{++}\right)=0.256_{-0.022}^{+0.024}$ (stat) \pm 0.014 (syst) ps. This value establishes the weakly-decaying nature of the $\Xi_{\mathrm{cc}}^{++}$state.

\section{Spectroscopy and searches}

\subsection{Observation of new states and decay modes}

Analyzing $4.5 \mathrm{fb}^{-1}$ of data at 7,8 , and $13 \mathrm{TeV}$, LHCb reported the observation of a new $\Xi_{\mathrm{b}}^{-}$ resonance, seen in the decay modes $\Lambda_{\mathrm{b}}^{0} \mathrm{~K}^{-}$and $\Xi_{\mathrm{b}}^{0} \pi^{-}$[22]. The observed structure has a mass $M=6226.9 \pm 2.0$ (stat) \pm 0.3 (syst) $\pm 0.2\left(\Lambda_{\mathrm{b}}^{0}\right) \mathrm{MeV}$ and a proper width $\Gamma=18.1 \pm 5.4$ (stat) \pm 1.8 (syst) $\mathrm{MeV}$. The new peak is interpreted as the excited $\Xi_{\mathrm{b}}(6227)^{-}$resonance, having bds quark 
content. Fig. 4 shows the distribution of the mass difference $M^{\star}\left(\Lambda_{\mathrm{b}}^{0} \mathrm{~K}^{-}\right)-M^{\star}\left(\Lambda_{\mathrm{b}}^{0}\right)$ in the $13 \mathrm{TeV}$ dataset, where the enhancement due to the new resonance is clearly visible on top of the smooth combinatorial background. The ratio between the branching fractions of the new particle in the two decay modes is estimated to be $\mathscr{B}\left(\Xi_{\mathrm{b}}(6227)^{-} \rightarrow \Lambda_{\mathrm{b}}^{0} \mathrm{~K}^{-}\right) / \mathscr{B}\left(\Xi_{\mathrm{b}}(6227)^{-} \rightarrow \Xi_{\mathrm{b}}^{0} \pi^{-}\right) \approx 1 \pm 0.5$.

The CMS collaboration recently released a preliminary study concerning excited $\mathrm{B}_{\mathrm{s}}^{0}$ states, where a new decay mode of the $\mathrm{B}_{\mathrm{s} 2}^{\star}(5840)^{0}$ resonance was observed for the first time [23]. The two $\mathrm{B}_{\mathrm{s}}^{0}$ excited states $\mathrm{B}_{\mathrm{s} 1}(5830)^{0}$ and $\mathrm{B}_{\mathrm{s} 2}^{\star}(5840)^{0}$ were originally observed by the CDF and D0 collaborations through their decays into $\mathrm{B}^{(\star)+} \mathrm{K}^{-}[24,25]$. Using $19.6 \mathrm{fb}^{-1}$ of pp data at $8 \mathrm{TeV}$, CMS studied the properties of these two states both in the $\mathrm{B}^{(\star)+} \mathrm{K}^{-}$and in the $\mathrm{B}^{(\star)}{ }^{\circ} \mathrm{K}_{\mathrm{S}}^{0}$ mode. The $\mathrm{B}_{\mathrm{s} 2}^{\star}(5840)^{0} \rightarrow \mathrm{B}^{0} \mathrm{~K}_{\mathrm{S}}^{0}$ decay was observed for the first time with a significance in excess of $6 \sigma$, and the first evidence of the $\mathrm{B}_{\mathrm{s} 1}(5830)^{0} \rightarrow \mathrm{B}^{\star 0} \mathrm{~K}_{\mathrm{S}}^{0}$ decay was obtained with a significance in excess of $3 \sigma$. The $\mathrm{B}^{0} \mathrm{~K}_{\mathrm{S}}^{0}$ invariant mass distribution is shown in Fig. 5. Several properties were measured. Among them, there is the first measurement of the mass difference between $\mathrm{B}^{\star 0}$ and $\mathrm{B}^{\star+}$ excited mesons $m_{\mathrm{B}^{\star 0}}-m_{\mathrm{B}^{\star+}}=0.91 \pm 0.24$ (stat) \pm 0.09 (syst) \pm 0.02 (PDG) MeV, and the first measurement of the branching fraction times cross section ratio:

$$
R_{\sigma}^{0} \equiv \frac{\sigma\left(\mathrm{pp} \rightarrow \mathrm{B}_{\mathrm{s} 1 \ldots)} \ldots \mathscr{B}\left(\mathrm{B}_{\mathrm{s} 1} \rightarrow \mathrm{B}^{\star 0} \mathrm{~K}_{\mathrm{S}}^{0}\right)\right.}{\sigma\left(\mathrm{pp} \rightarrow \mathrm{B}_{\mathrm{s} 2}^{\star} \ldots\right) \times \mathscr{B}\left(\mathrm{B}_{\mathrm{s} 2}^{\star} \rightarrow \mathrm{B}^{0} \mathrm{~K}_{\mathrm{S}}^{0}\right)}=0.266 \pm 0.079(\text { stat }) \pm 0.063 \text { (syst) } .
$$

Another recent observation of a new decay mode comes from LHCb and concerns the $\Lambda_{\mathrm{b}}^{0}$ baryon. The 4-body decay mode $\Lambda_{\mathrm{b}}^{0} \rightarrow \Lambda_{\mathrm{c}}^{+} \mathrm{p} \overline{\mathrm{p}} \pi^{-}$was observed for the first time using $3 \mathrm{fb}^{-1}$ of pp data taken at 7 and $8 \mathrm{TeV}$ [26]. The $\Lambda_{\mathrm{b}}^{0} \rightarrow \Lambda_{\mathrm{c}}^{+} \mathrm{p} \overline{\mathrm{p}} \pi^{-}$invariant mass distribution measured by LHCb can be seen in Fig. 6. The analysis of the $\Lambda_{\mathrm{c}}^{+} \pi^{-}$invariant mass distribution showed that this decay can proceed through the intermediate production of $\Sigma_{\mathrm{c}}(2455)^{0}$ and $\Sigma_{\mathrm{c}}^{\star}(2520)^{0}$ resonances, with branching fraction ratios between the resonant modes and the total 4-body mode of $0.089 \pm$ $0.015 \pm 0.006$ and $0.119 \pm 0.020 \pm 0.014$, respectively. Possible di-baryon resonances were also searched for in the $\Lambda_{\mathrm{c}}^{+} \pi^{-} \mathrm{p}$ invariant mass spectrum, but no evidence of peaking structures was found.

Table 1: The B hadron lifetimes measured by CMS on $8 \mathrm{TeV}$ data [18]. The last column shows the world averages from PDG [19].

\begin{tabular}{|l|l|l|}
\hline Channel & CMS result $[\mu \mathbf{m}]$ & PDG $[\mu \mathbf{m}]$ \\
\hline $\mathrm{B}^{0}$ & $454.1 \pm 1.4($ stat $) \pm 1.7($ syst $)$ & $455.7 \pm 1.2$ \\
$\mathrm{~B}_{\mathrm{s}}^{0} \rightarrow \mathrm{J} / \psi \pi^{+} \pi^{-}$ & $502.7 \pm 10.2($ stat $) \pm 3.4($ syst $)$ & $482.4 \pm 3.0$ \\
$\mathrm{~B}_{\mathrm{s}}^{0} \rightarrow \mathrm{J} / \psi \phi(1020)^{0}$ & $443.9 \pm 2.0($ stat $) \pm 1.5($ syst $)$ & $443.4 \pm 3.6$ \\
$\Lambda_{\mathrm{b}}^{0}$ & $442.9 \pm 8.2($ stat $) \pm 2.8($ syst $)$ & $440.7 \pm 3.0$ \\
$\mathrm{~B}_{\mathrm{c}}^{+}$ & $162.3 \pm 7.8($ stat $) \pm 4.2($ syst $) \pm 0.1\left(\mathrm{~B}^{+}\right)$ & $152.0 \pm 2.7$ \\
\hline
\end{tabular}

\subsection{Other searches for new and exotic hadrons}

LHCb recently submitted an article where the search for exotic b $b \bar{b} \bar{b}$ tetraquarks is performed on $6 \mathrm{fb}^{-1}$ of data at 7,8 , and $13 \mathrm{TeV}$ [27]. The $\mathrm{X}_{\mathrm{b} \bar{b} \mathrm{~b} \bar{b}}$ states are looked for in the $\mathrm{Y}(1 \mathrm{~S}) \mu^{+} \mu^{-}$ decay mode, with $\mathrm{Y}(1 \mathrm{~S})$ decaying to $\mu^{+} \mu^{-}$. As can be seen in Fig. 7, no significant excess is observed in the $m\left(\mathrm{X}_{\mathrm{b} \bar{b} \bar{b} \bar{b}}\right)$ mass range $[16,26] \mathrm{GeV}$. Limits on the production cross section times 
branching fraction of the hypothetical resonance are set as a function of $m\left(\mathrm{X}_{\mathrm{b} \bar{b} \mathrm{~b}}\right)$ in the restricted range $[17.5,20] \mathrm{GeV}$. The results are compatible with the background-only hypothesis, with the largest deviation, having $\approx 2.5 \sigma$ local significance, seen at $m\left(\mathrm{X}_{\mathrm{b} \bar{b} \mathrm{~b}}\right)=19.35 \mathrm{GeV}$.

The evidence reported a few years ago by the $\mathrm{D} 0$ experiment of an enhancement in the $\mathrm{B}_{\mathrm{s}}^{0} \pi^{ \pm}$ mass spectrum [28], called X (5568), has generated a large amount of further research. Three LHC experiments (ATLAS, CMS, and LHCb) have looked for this resonance in their data, finding no evidence for this hypothetical state $[29,30,31]$. Stringent limits to $\rho_{\mathrm{X}}$, defined as the ratio between the number of $\mathrm{B}_{\mathrm{s}}^{0}$ coming from the $\mathrm{X}(5568)$ decay with respect to the total $\mathrm{B}_{\mathrm{s}}^{0}$ production, were then set as $\rho_{\mathrm{X}}<1.5 \%$ at $95 \%$ confidence level, CL (ATLAS), $\rho_{\mathrm{X}}<1.1 \%$ at $95 \% \mathrm{CL}$ (CMS), and $\rho_{\mathrm{X}}<1.2 \%$ at $95 \% \mathrm{CL}(\mathrm{LHCb})$. These limits are much lower than the value of $\rho_{\mathrm{X}}$ reported by the D0 experiment, $\rho_{\mathrm{X}}=(8.6 \pm 1.9 \pm 1.4) \%$.

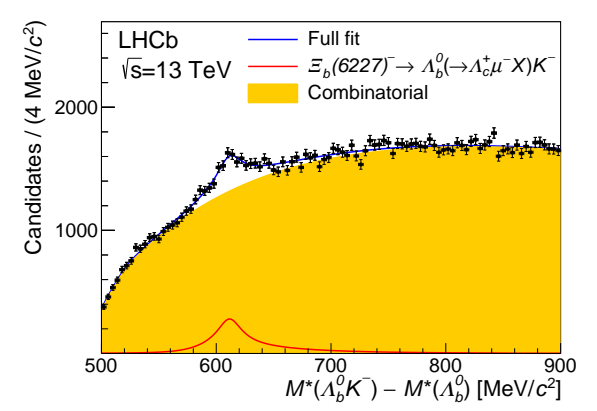

Figure 4: The invariant mass difference for the $\Lambda_{\mathrm{b}}^{0} \mathrm{~K}^{-}$channel measured by $\mathrm{LHCb}$ on $13 \mathrm{TeV}$ data. The peak due to the new $\Xi_{\mathrm{b}}(6227)^{-}$resonance is clearly visible on top of the combinatorial background [22].

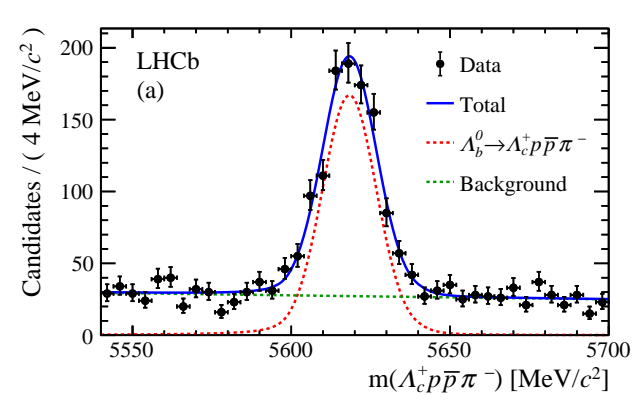

Figure 6: The $\Lambda_{c}^{+} p \bar{p} \pi^{-}$invariant mass distribution measured by $\mathrm{LHCb}$, showing the peak from the newly discovered $\Lambda_{\mathrm{b}}^{0} \rightarrow \Lambda_{\mathrm{c}}^{+} \mathrm{p} \overline{\mathrm{p}} \pi^{-}$decay on top of the flat background [26].

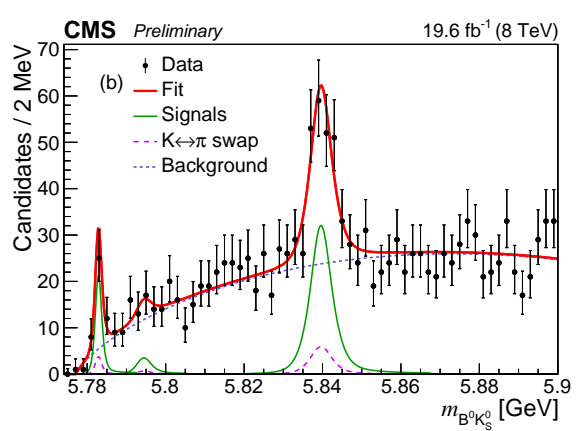

Figure 5: The $\mathrm{B}^{0} \mathrm{~K}_{\mathrm{S}}^{0}$ invariant mass distribution measured by $\mathrm{CMS}$ on $8 \mathrm{TeV}$ data. The decays $\mathrm{B}_{\mathrm{s} 2}^{\star}(5840)^{0} \rightarrow \mathrm{B}^{0} \mathrm{~K}_{\mathrm{S}}^{0}$ and $\mathrm{B}_{\mathrm{s} 1}(5830)^{0} \rightarrow \mathrm{B}^{\star 0} \mathrm{~K}_{\mathrm{S}}^{0}$ are visible as the two enhancements at $5.84 \mathrm{GeV}$ and $5.78 \mathrm{GeV}$, respectively [23].

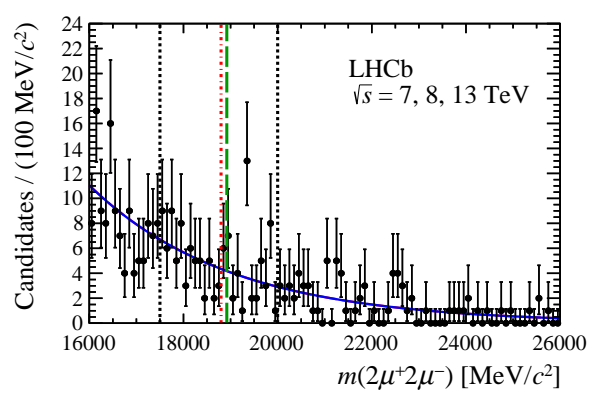

Figure 7: The 4- $\mu$ invariant mass distribution measured by $\mathrm{LHCb}$ in the 7,8 , and $13 \mathrm{TeV}$ datasets in the search for $b \bar{b} b \bar{b}$ resonances. The black lines show the range where $\sigma \times \mathscr{B}$ limits are set [27].

Another search for which disagreeing results are found by different experiments concerns possible excited $\mathrm{B}_{\mathrm{c}}^{+}$states. In 2014, ATLAS had reported the first observation of a narrow resonance in the $\mathrm{B}_{\mathrm{c}}^{+} \pi^{+} \pi^{-}$invariant mass spectrum, interpreted as the $\mathrm{B}_{\mathrm{c}}^{+}(2 \mathrm{~S})$ state [32]. The peak was seen in both $7 \mathrm{TeV}$ and $8 \mathrm{TeV}$ data (see Fig. 8 left, for the latter), with a mass $M=6842 \pm 4 \pm 5 \mathrm{MeV}$ and a 
global significance of $5.2 \sigma$. Recently, the $\mathrm{B}_{\mathrm{c}}^{+}(2 \mathrm{~S})$ state has been the object of a search performed by $\mathrm{LHCb}$ on $2 \mathrm{fb}^{-1}$ of $8 \mathrm{TeV}$ pp data [33]. In this new work, no evidence for the state claimed by ATLAS is seen (see Fig. 8 right). Upper limits to the ratio of the $\mathrm{B}_{\mathrm{c}}^{+}(2 \mathrm{~S}) \sigma \times \mathscr{B}$ with respect to the $\mathrm{B}_{\mathrm{c}}^{+}$cross section were set by $\mathrm{LHCb}$ as a function of the invariant mass, ranging between $\sim 0.02$ and $\sim 0.14$ at $95 \% \mathrm{CL}$. Given this contrasting result, the question of the confirmation of ATLAS observation remains open.
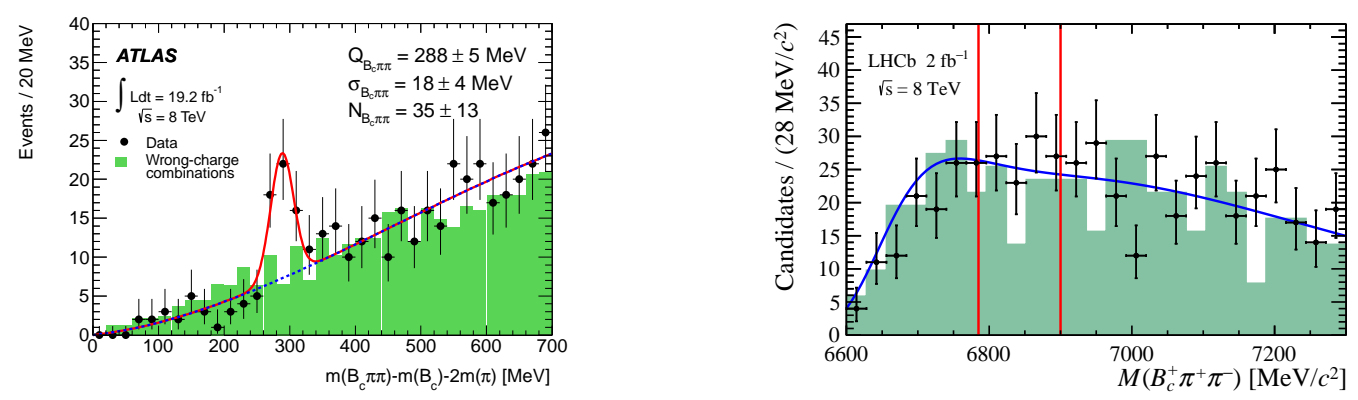

Figure 8: The invariant mass region near the $\mathrm{B}_{\mathrm{c}}^{+}(2 \mathrm{~S})$ resonance measured by ATLAS (left) and LHCb (right) on $8 \mathrm{TeV}$ pp data [32, 33].

\section{References}

[1] ALICE collaboration, The ALICE experiment at the CERN LHC, JINST 3 (2008) S08002.

[2] ATLAS collaboration, The ATLAS Experiment at the CERN Large Hadron Collider, JINST 3 (2008) S08003.

[3] CMS collaboration, The CMS experiment at the CERN LHC, JINST 3 (2008) S08004.

[4] LHCB collaboration, The LHCb Detector at the LHC, JINST 3 (2008) S08005.

[5] CMS collaboration, Measurement of quarkonium production cross sections in pp collisions at $\sqrt{s}=13 \mathrm{TeV}$, Physics Letters B $\mathbf{7 8 0}$ (2018) 251 [arXiv:1710.11002].

[6] LHCB collaboration, Measurement of $\Upsilon$ production in pp collisions at $\sqrt{s}=13 \mathrm{TeV}$, JHEP 07 (2018) 134 [arXiv:1804.09214].

[7] G. T. Bodwin, E. Braaten, G. P. Lepage, Rigorous QCD analysis of inclusive annihilation and production of heavy quarkonium, Phys. Rev. D 51 (1995) 1125 [arXiv: hep-ph/9407339].

[8] ATLAS collaboration, Measurement of the differential cross-sections of prompt and non-prompt production of $\mathrm{J} / \psi$ and $\psi(2 \mathrm{~S})$ in pp collisions at $\sqrt{s}=7$ and $8 \mathrm{TeV}$ with the ATLAS detector, Eur. Phys. J. C 76 (2016) 283 [arXiv:1512.03657].

[9] J. P. Lansberg, On the mechanisms of heavy-quarkonium hadroproduction, Eur. Phys. J. C 61 (2009) 693 [arXiv:0811.4005].

[10] ALICE collaboration, Measurement of the inclusive $\mathrm{J} / \psi$ polarization at forward rapidity in $p p$ collisions at $\sqrt{s}=8 \mathrm{TeV}$, submitted to Eur. Phys. J. C [arXiv:1805.04374].

[11] CMS collaboration, Observation of the $\chi_{\mathrm{b} 1}(3 \mathrm{P})$ and $\chi_{\mathrm{b} 2}(3 \mathrm{P})$ and measurement of their masses, Phys. Rev. Lett. 121 (2018) 092002 [arXiv: 1805.11192].

[12] ATLAS collaboration, Observation of a New $\chi_{\mathrm{b}}$ State in Radiative Transitions to $\mathrm{Y}(1 \mathrm{~S})$ and $\mathrm{Y}(2 \mathrm{~S})$ at ATLAS, Phys. Rev. Lett. 108 (2012) 152001 [arXiv:1112.5154]. 
[13] ALICE collaboration, $\Lambda_{\mathrm{c}}^{+}$production in pp collisions at $\sqrt{s}=7 \mathrm{TeV}$ and in $p$-Pb collisions at $\sqrt{s}=5.02 \mathrm{TeV}$, JHEP 04 (2018) 108 [arXiv: 1712.09581$].$

[14] ALICE collaboration, First measurement of $\Xi_{\mathrm{c}}^{0}$ production in pp collisions at $\sqrt{s}=7 \mathrm{TeV}$, Phys. Lett. B 781 (2018) 8 [arXiv:1712.04242].

[15] LHCB collaboration, Measurement of $\mathrm{D}_{\mathrm{s}}^{ \pm}$production asymmetry in pp collisions at $\sqrt{s}=7$ and 8TeV, JHEP 08 (2018) 008 [arXiv: 1805 . 09869].

[16] T. Sjöstrand, S. Mrenna, P. Skands, A brief introduction to Pythia 8.1, "Comp. Phys. Comm." 178 (2008) 852.

[17] LHCB collaboration, Measurement of the $\mathrm{D}_{\mathrm{s}}^{+}-\mathrm{D}_{\mathrm{s}}^{-}$production asymmetry in $7 \mathrm{TeV}$ pp collisions, Phys. Lett. B 713 (2012) 186 [arXiv:1205.0897].

[18] CMS collaboration, Measurement of b hadron lifetimes in pp collisions at $\sqrt{s}=8 \mathrm{TeV}$, Eur. Phys. $J$. C 78 (2018) 457 [arXiv:1710.08949].

[19] C. Patrignani et al., [Particle Data Group], Review of particle physics, Chinese Physics C 40 (2016) 100001.

[20] LHCв collaboration, Observation of the Doubly Charmed Baryon $\Xi_{c c}^{++}$, Phys. Rev. Lett. 119 (2017) 112001 [arXiv:1707-01621].

[21] LHCB collaboration, Measurement of the Lifetime of the Doubly Charmed Baryon $\Xi_{\mathrm{cc}}^{++}$, Phys. Rev. Lett. 121 (2018) 052002 [arXiv: 1806.02744 ].

[22] LHCB collaboration, Observation of a New $\Xi_{\mathrm{b}}^{-}$Resonance, Phys. Rev. Lett. 121 (2018) 072002 [arXiv:1805.09418].

[23] CMS collaboration, Observation of the $\mathrm{B}_{\mathrm{s} 2}^{\star}(5840)^{0} \rightarrow \mathrm{B}^{0} \mathrm{~K}_{\mathrm{S}}^{0}$ decay and studies of excited $\mathrm{B}_{\mathrm{s}}^{0}$ mesons in proton-proton collisions at $\sqrt{s}=8 \mathrm{TeV}, C M S-P A S-B P H-16-003$ (2018) submitted to Eur. Phys. J. C.

[24] CDF collaboration, Observation of Orbitally Excited $\mathrm{B}_{\mathrm{s}}$ Mesons, Phys. Rev. Lett. 100 (2008) 082001 [arXiv:0710.4199].

[25] D0 collaboration, Observation and Properties of the Orbitally Excited $\mathrm{B}_{\mathrm{s} 2}^{\star}$ Meson, Phys. Rev. Lett. 100 (2008) 082002 [arXiv: 0711.0319 ].

[26] LHCB collaboration, Observation of the decay $\Lambda_{\mathrm{b}}^{0} \rightarrow \Lambda_{\mathrm{c}}^{+} \mathrm{p} \overline{\mathrm{p}} \pi^{-}$, Phys. Lett. B 784 (2018) 101 [arXiv:1804.09617].

[27] LHCB collaboration, Search for beautiful tetraquarks in the $\mathrm{Y}(1 \mathrm{~S}) \mu^{+} \mu^{-}$invariant-mass spectrum, LHCb-PAPER-2018-027 (2018) submitted to JHEP [arXiv:1806.09707].

[28] D0 collaboration, Evidence for $a \mathrm{~B}_{\mathrm{s}}^{0} \pi^{ \pm}$State, Phys. Rev. Lett. 117 (2016) 022003.

[29] ATLAS collaboration, Search for a Structure in the $\mathrm{B}_{\mathrm{s}}^{0} \pi^{ \pm}$Invariant Mass Spectrum with the ATLAS Experiment, Phys. Rev. Lett. 120 (2018) 202007 [arXiv: 1802.01840 ].

[30] CMS collaboration, Search for the $\mathrm{X}$ (5568) State Decaying into $\mathrm{B}_{\mathrm{s}}^{0} \pi^{ \pm}$in Proton-Proton Collisions at $\sqrt{s}=8 \mathrm{TeV}$, Phys. Rev. Lett. 120 (2018) 202005 [arXiv: 1712.06144 ].

[31] LHCB collaboration, Search for Structure in the $\mathrm{B}_{\mathrm{s}}^{0} \pi^{ \pm}$Invariant Mass Spectrum, Phys. Rev. Lett. 117 (2016) 152003 [arXiv:1608.00435].

[32] ATLAS collaboration, Observation of an Excited $\mathrm{B}_{\mathrm{c}}^{ \pm}$Meson State with the ATLAS Detector, Phys. Rev. Lett. 113 (2014) 212004 [arXiv:1407.1032].

[33] LHCв collaboration, Search for excited $\mathrm{B}_{\mathrm{c}}^{+}$states, JHEP 08 (2018) 138 [arXiv:1712. 04094 ]. 